\title{
Simple, Amplified, and Multiplexed Detection of MicroRNAs Using Time-Gated FRET and Hybridization Chain Reaction
}

\author{
Jiajia Guo, Carlos Mingoes, Xue Qiu, and Niko Hildebrandt*i( \\ NanoBioPhotonics (nanofret.com), Institute for Integrative Biology of the Cell (I2BC), Université Paris-Saclay, Université \\ Paris-Sud, CNRS, CEA, 91400 Orsay, France
}

\section{Supporting Information}

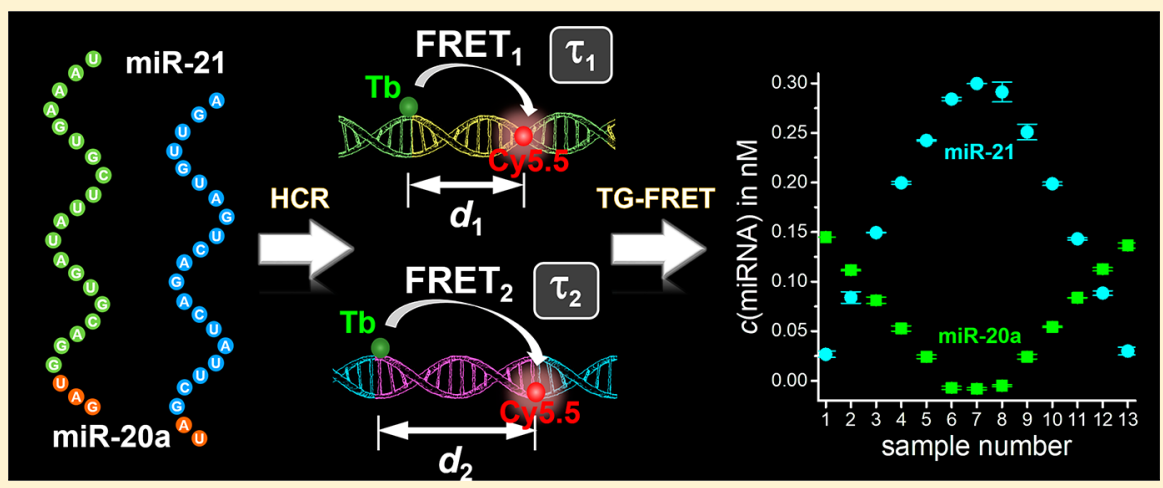

ABSTRACT: The hybridization chain reaction (HCR) is a simple and sensitive method for quantifying nucleic acids. Current approaches cannot combine a washing-free sensing format with multiplexed target quantification at low concentrations, which would be highly desirable for detection both in solution and in situ. Here, we demonstrate the implementation of time-gated Förster resonance energy transfer (TG-FRET) between terbium donors and dye acceptors into HCR for multiplexed quantification of microRNAs (miR-20a and miR-21) and their DNA analogues. HCR-TG-FRET provided washing-free nucleic acid quantification with very low limits of detection down to 240 amol (1.7 pM) of microRNA and 123 amol (0.88 pM) of DNA. Efficient distinction from very homologous microRNAs demonstrated high target specificity. Multiplexing with a single measurement, a single excitation wavelength, and a single FRET pair allowed for a simultaneous quantification of miR-20a and miR-21 at concentrations between 30 and $300 \mathrm{pM}$ from the same sample. HCR-TG-FRET showed similar performance for serum-free and serum-containing samples without the use of RNase inhibitors. Our results present a significant improvement in current HCR approaches regarding simplicity, sensitivity, and multiplexing. The versatile diagnostic performance of HCR-TGFRET even in challenging biological environments presents an important advantage for advanced nucleic acid biosensing.

$I^{s}$ sothermal amplification of nucleic acids is a frequently used approach for detecting low concentrations of DNA or RNA. ${ }^{1}$ Many different exponential and linear nucleic acid amplification and signal amplification (without the generation of new nucleic acid products) strategies have been developed over the last few decades. ${ }^{1}$ One of the most simple isothermal signal amplification methods is the hybridization chain reaction (HCR), because it is completely enzyme free. ${ }^{2} \mathrm{HCR}$ has been widely used to construct various biosensing platforms, including the detection of DNA, ${ }^{3,4}$ micro-RNA (miRNA), ${ }^{5,6}$ messenger-RNA (mRNA), ${ }^{7,8}$ proteins, ${ }^{9,10}$ and cells. ${ }^{11}$ Moreover, HCR technologies have been significantly advanced for both multiplexed imaging and quantitation of endogenous nucleic acids. ${ }^{12-15}$

One possibility for multiplexed detection of different targets by HCR is the application of fluorophores with distinct emission colors. For example, color multiplexing of fluorescent dyes was used for imaging of five different mRNA targets. ${ }^{7}$ Such spectral multiplexing has significant limitations due to the requirement of different excitation wavelengths and strongly overlapping fluorescence spectra. In particular, the quantitation of low target concentrations requires spectral crosstalk correction. ${ }^{16,17}$ Another multiplexing approach is target-specific signal generation. For example, combining several target-specific HCR probes on the same gold nanoparticle with controlled signal generation by specific HCR initiators was used for the quantification of three different miRNA targets. ${ }^{14}$ Because the same optical signal (plasmon absorption band of the gold nanoparticles) was used for all targets, their distinction required one individual HCR amplification and detection step per target. $^{14}$

The simplicity of HCR can be improved by attaching FRET (Förster resonance energy transfer) donor-acceptor pairs to the HCR probes. ${ }^{3,8,11,18-25}$ With this approach, HCR amplification leads to double-stranded DNA concatemers with many donors and acceptors in close proximity. Only HCR amplification can

Received: December 4, 2018

Accepted: January 18, 2019

Published: January 18, 2019 
Table 1. DNA and miRNA Oligonucleotides Used in the Study ${ }^{a}$

\begin{tabular}{|l|l|}
\hline Name & Sequence 5'-3' \\
\hline has-miR-20a & UAAAGUGCUUAUAGUGCAGGUAG \\
\hline has-miR-20b & CAAAGUGCUCAUAGUGCAGGUAG \\
\hline has-miR-21 & UAGCUUAUCAGACUGAUGUUGA \\
\hline ssDNA-20a & 'TAAAGTGCTTATAGTGCAGGTAG \\
\hline ssDNA-20b & CAAAGTGCTCATAGTGCAGGTAG \\
\hline ssDNA-21 & TAGCTTATCAGACTGATGTTGA \\
\hline Hairpin probe 1 (H1) & CCTGCACTATAAGCACTTTATAGCCTTAAAGTGCTTATAG-Tb \\
\hline Hairpin probe 2 (H2) & TAAAGTGCT (CY5.5) TATAGTGCAGGCTATAAGCACTTTAAGGCTA \\
\hline Hairpin probe 3 (H3) & Tb-CAGACTGATGTTGATAGCCTTCAACATCAGTCTGATAAGC \\
\hline Hairpin probe 4 (H4) & AGGCTATCAACATCAGTCTGGCTTATCAGACTGATGTTGA-CY5.5 \\
\hline
\end{tabular}

${ }^{a}$ Mismatches between miR-20a and miR-20b are shown in red, Tb functionalizations in green, and Cy5.5 functionalizations in blue.

lead to FRET, whereas free HCR FRET probes are separated and cannot engage in energy transfer due to the spatial separation of donor and acceptor. Thus, the FRET signal is amplification-specific and target-specific and washing steps (separation of free HCR probes from amplified HCR product) are not required, which significantly improves the simplicity and duration of the experiment. Although HCR-FRET has been demonstrated for both imaging in cells and quantification in solution, its combination with multiplexing has not been realized and low-picomolar limits of detection (LODs) could only be accomplished by double-step amplification. ${ }^{22}$

Recently, we developed various different approaches for sensitive and multiplexed quantification of nucleic acids using time-gated (TG) FRET from $\mathrm{Tb}$ complexes as donors to dyes and quantum dots as acceptors. ${ }^{17,26-29}$ TG-FRET (also known as HTRF or TR-FRET) $)^{30}$ exploits the long photoluminescence (PL) lifetimes of lanthanides for autofluorescence-free detection of FRET-sensitized acceptor PL intensities in a delayed (several microseconds after pulsed excitation) detection window. ${ }^{31}$ In particular, we could demonstrate higher-order multiplexing by using both the temporal and spectral components of TG-FRET, limits of detection down to $40 \mathrm{zmol}$ of DNA, and precise quantification of endogenous miRNAs from human cells, tissues, and blood. ${ }^{27,29}$ Merging the multiplexing capabilities, background-free detection, and separation-free assay format of TG-FRET with the enzyme-free amplification of HCR has the potential to significantly advance nucleic acid biosensing.

To demonstrate the feasibility of simple and sensitive HCR nucleic acid multiplexing, we selected two different miRNAs, miR-21 and miR-20a, both of which were found to be mainly upregulated in different cancers (220 and 60 miRNA-cancer relations for hsa-miR-21 and hsa-miR-20a, respectively, as reported in the miRCancer database). ${ }^{32,33}$ Many amplified miRNA detection methods have been reported, including reverse transcription quantitative polymerase chain reaction (RT-qPCR), ${ }^{34,35}$ rolling circle amplification (RCA), ${ }^{36,37}$ and strand displacement amplification (SDA). ${ }^{38,39}$ Despite very high sensitivities and specificities of such techniques, their application is still relatively complex. All of those techniques require enzymes and in some cases RNase inhibitors. ${ }^{40,41}$ Other drawbacks include time-consuming procedures, the necessity of temperature cycling, and exponential amplification, which is usually less accurate for quantitative measurements in comparison to linear amplification. Here, we implement a Tb- dye TG-FRET pair into HCR probes to demonstrate temporally multiplexed enzyme-free quantification of two different miRNAs with a single measurement, a single excitation wavelength, and a single detection channel. Both very low LODs (down to a few hundred attomoles) and high selectivity against very homologous miRNAs (dual-nucleotide variants) demonstrated the unprecedented performance of our multiplexed HCR-TGFRET assay.

\section{MATERIALS AND METHODS}

Materials. Lumi4-Tb-NHS ( $\mathrm{Tb}$ ) was provided by Lumiphore Inc. Cyanine5.5 NHS ester was purchased from Lumiprobe. All oligonucleotides were purchased from Eurogentec. Nonmodified oligonucleotides were purified by the supplier (cartridge gold desalting method). Cy5.5-modified oligonucleotides were purified with HPLC. All sequences and modifications of nucleic acids used in this study are summarized in Table 1. Both Cyanine5.5 (Lumiprobe) and Cy5.5 (GE Healthcare Cy dye used by Eurogentec for functionalization) are referred to as Cy5.5 because of their very similar photophysical properties and chemical structures (Cyanine5.5 NHS ester can replace NHS esters of Cy5.5). ${ }^{42}$ Tris(hydroxymethyl)aminomethane, bovine serum albumin, and HEPES were purchased from Sigma-Aldrich. $\mathrm{NaCl}$ was purchased from Duchefa. All chemicals were used as received. Water was purified by MAXIMA (USF Elga, U.K.). Zeba Spin Desalting Columns ( $7 \mathrm{kDa}$ MWCO) were purchased from Thermo Fisher Scientific.

Tb-DNA Conjugates. Tb-DNA conjugates (hairpin probes 1 and 3 ) were obtained by mixing Lumi4-Tb-NHS (lyophilized product was dissolved in anhydrous DMF to a final concentration of $8 \mathrm{mM}$ stock solution) in concentration excess to amino-functionalized oligonucleotides in $100 \mathrm{mM}$ carbonate buffer at $\mathrm{pH}$ 9.0. The mixtures were incubated overnight at $4{ }^{\circ} \mathrm{C}$. The Tb-DNA conjugates were purified three times by Zeba Spin Desalting Columns (7 kDa MWCO). Complete bioconjugation of DNA with $\mathrm{Tb}(\sim 100 \%)$ was verified by absorbance measurements at $340 \mathrm{~nm}$ for $\mathrm{Tb}$ and $260 \mathrm{~nm}$ for DNA.

Dye-DNA Conjugates. Dye-DNA conjugates were purchased at Eurogentec with 3'-terminal Cy5.5 (hairpin probe 4) or prepared by mixing Cyanine5.5 NHS ester (lyophilized product dissolved in anhydrous DMF to a final concentration of $16.6 \mathrm{mM}$ stock solution) in concentration excess to aminofunctionalized oligonucleotides in $100 \mathrm{mM}$ carbonate buffer at 
pH 9.0 (hairpin probe 2). The prepared mixtures were incubated overnight at $4{ }^{\circ} \mathrm{C}$ and then purified three times by Zeba Spin Desalting Columns (7 kDa MWCO). Complete bioconjugation of DNA with Cy5.5 ( 100\%) was provided by Eurogentec (hairpin probe 4) or verified by absorbance measurements at $684 \mathrm{~nm}$ for Cy5.5 and $260 \mathrm{~nm}$ for DNA (hairpin probe 2).

Spectroscopic Characterization. Absorption spectra (Lambda 35 UV/vis System, PerkinElmer) and emission spectra (Xenius, SAFAS) for Tb- and dye-functionalized DNA were recorded in HEPES (100 mM, pH 7.4). All spectra are shown in Figure 1. Both Cy5.5 dyes have very similar spectra, use the same

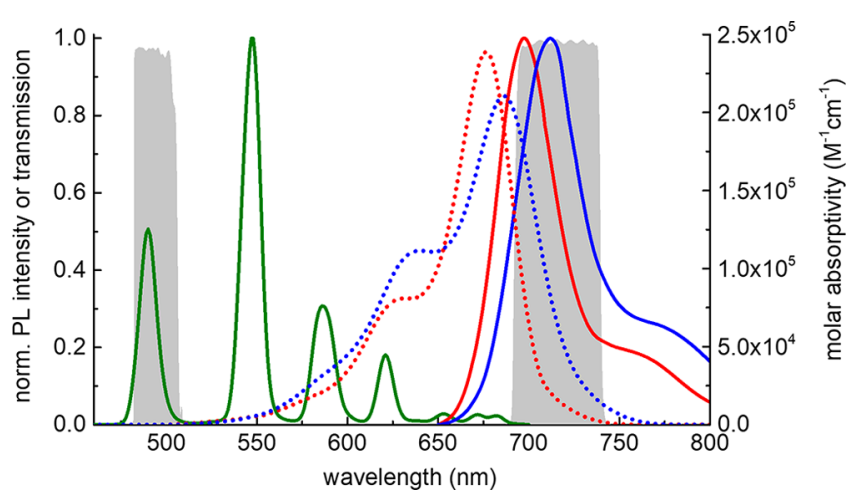

Figure 1. Photophysical properties of the Tb-Cy5.5 FRET pair. Absorbance spectra (dotted, right axis) and PL emission spectra (line, left axis) of Cy5.5 (red for Cy5.5 from Eurogentec and blue for Cy5.5 from Lumiprobe). PL emission spectrum of $\mathrm{Tb}$ (green, left axis). Absorption spectrum of $\mathrm{Tb}$ not shown (maximum molar absorptivity of $26000 \mathrm{M}^{-1} \mathrm{~cm}^{-1}$ at $\left.340 \mathrm{~nm}\right)$. Transmission spectra of optical band-pass filters (494/20 nm (Semrock) for the Tb detection channel and 716/40 $\mathrm{nm}$ (Semrock) for the Cy5.5 detection channel) with $494 \pm 12$ and 716 $\pm 22 \mathrm{~nm}$ band pass wavelength ranges, respectively (gray, left axis). For PL spectra, excitation wavelengths were $340 \pm 2.5 \mathrm{~nm}$ for $\mathrm{Tb}$ and $630 \pm$ $2.5 \mathrm{~nm}$ for Cy5.5.

PL detection bandpass filter, and lead to almost identical Förster distances. A Förster distance (the donor-acceptor distance for which FRET is $50 \%$ efficient) for the Tb-Cy5.5 FRET pair of $R_{0}$ $=5.8 \pm 0.2 \mathrm{~nm}$ was calculated using eq 1 :

$$
R_{0}=0.0211\left(\kappa^{2} \Phi_{\mathrm{D}^{n}}{ }^{-4} J\right)^{1 / 6} \mathrm{~nm}
$$

An orientation factor of $\kappa^{2}=2 / 3$ was used because of the random orientation of donor and acceptor during the FRET time (dynamic averaging), which is well justified by the long PL lifetime of the $\mathrm{Tb}$ donors and their unpolarized emission (fast isotropic rotation). A refractive index of $n=1.35$ (aqueous solution) was used. The Tb-centered quantum yield was $\Phi_{\mathrm{D}}=$ $0.78 \pm 0.05$, as calculated by $\Phi_{\mathrm{D}}=\tau_{\mathrm{D}} / \tau_{\mathrm{n}}$ with $\tau_{\mathrm{D}}=2.70 \pm 0.08$ $\mathrm{ms}$ (PL lifetime of $\mathrm{Tb}$ in our experiments) and $\tau_{\mathrm{n}}=3.45 \pm 0.12$ ms (intrinsic PL lifetime of $\mathrm{Tb}$, as measured in $\mathrm{D}_{2} \mathrm{O}$ at room temperature). The spectral overlap integral $J$ was calculated by eq 2:

$$
J=\int_{450 \mathrm{~nm}}^{700 \mathrm{~nm}} I_{\mathrm{D}}(\lambda) \varepsilon_{\mathrm{A}}(\lambda) \lambda^{4} \mathrm{~d} \lambda
$$

where $I_{\mathrm{D}}(\lambda)$ is the emission intensity from the area-normalized (to unity) emission spectrum of the Tb donor and $\varepsilon_{\mathrm{A}}(\lambda)$ is the molar absorptivity of the acceptor.

HCR-FRET miRNA/ssDNA Single-Target Assays. MicroRNA assays are shown and discussed in the main text and the
Supporting Information. All ssDNA assays (DNA target with analogous sequence to the miRNA targets; see Table 1) are shown and discussed in the Supporting Information. Stock solutions $(30 \mathrm{nM})$ of hairpin probes were prepared in DNA hybridization buffer $(20 \mathrm{mM}$ Tris-Cl, $500 \mathrm{mM} \mathrm{NaCl}, 0.1 \% \mathrm{BSA}$, $\mathrm{pH}$ 8.0). Hairpin probes were heated to $95^{\circ} \mathrm{C}$ for $2 \mathrm{~min}$ and then cooled to room temperature for $2 \mathrm{~h}$ to form hairpin structures. The annealed hairpin probes were stored in the refrigerator $(4$ $\left.{ }^{\circ} \mathrm{C}\right)$. For the detection of the target, $10 \mu \mathrm{L}$ of $\mathrm{H} 1 / \mathrm{H} 3$ and $10 \mu \mathrm{L}$ of target miRNA/ssDNA at different concentrations were placed in $0.5 \mathrm{~mL}$ Eppendorf tubes. After incubation at room temperature for $30 \mathrm{~min}, 10 \mu \mathrm{L}$ of $\mathrm{H} 2 / \mathrm{H} 4$ was added and the mixture was further incubated for $60 \mathrm{~min}$. Then, $120 \mu \mathrm{L}$ of hybridization buffer was added to the solution to obtain a sufficient assay volume. From the total volume of $150 \mu \mathrm{L}, 140 \mu \mathrm{L}$ was transferred into black 96-well microtiter plates for miRNA/ ssDNA TG-FRET assays and PL decay analysis. TG-FRET assays were performed on a clinical immunofluorescence plate reader "KRYPTOR compact plus" (Thermo Fisher Scientific) with time gated $(0.1-0.9 \mathrm{~ms}) \mathrm{PL}$ intensity $\left(I^{\mathrm{TG}}\right)$ detection for obtaining target concentration dependent FRET ratios $\left(F_{\mathrm{R}}=\right.$ $\left.I(\mathrm{Cy} 5.5)^{\mathrm{TG}} / I(\mathrm{~Tb})^{\mathrm{TG}}\right)$. PL decays were recorded on a fluorescence lifetime plate reader (Edinburgh Instruments). For both plate readers, PL signals of $\mathrm{Tb}$ and $\mathrm{Cy} 5.5$ were measured in dedicated detection channels. Spectral distinction was accomplished by optical band pass filters (cf. Figure 1 for filter transmission spectra) for the $\mathrm{Tb}$ detection channel (Semrock 492/20 nm) and for the Cy5.5 detection channel (Semrock 716/40 nm).

HCR-FRET miRNA/ssDNA Duplexed Assays. The preparation of the assays was the same as for single-target assays but with $\mathrm{H} 1+\mathrm{H} 3$ (duplexed) instead of $\mathrm{H} 1 / \mathrm{H} 3$ (single) and $\mathrm{H} 2+\mathrm{H} 4$ (duplexed) instead of $\mathrm{H} 2 / \mathrm{H} 4$ (single). Assays and PL decay analysis were both performed using a fluorescence lifetime plate reader (Edinburgh Instruments) with 4000 detection bins of 2 $\mu$ s integration time and pulsed nitrogen laser (MNL 100, LTB Lasertechnik Berlin) excitation $(337.1 \mathrm{~nm}, 20 \mathrm{~Hz})$. TG-FRET assays used two TG PL detection windows $\left(\mathrm{TG}_{1}, 0.1-1.0 \mathrm{~ms}\right.$; $\mathrm{TG}_{2}, 2.0-3.0 \mathrm{~ms}$ ) for FRET-ratio calculation. TG PL intensities $\left(I^{\mathrm{TG}}\right)$ of $\mathrm{Cy} 5.5$ and $\mathrm{Tb}$ were determined at constant concentrations of hairpin probes and increasing concentrations of targets. These TG PL intensities were then used to calculate a background-subtracted FRET ratio $\left(F_{\mathrm{R}-\mathrm{BG}}\right.$, eq 3$)$ for each target concentration $c=x$.

$$
F_{\mathrm{R}-\mathrm{BG}}=\frac{I_{\mathrm{C} y 5.5}^{\mathrm{TG}}(c=x)}{I_{\mathrm{Tb}}^{\mathrm{TG}}(c=x)}-\frac{I_{\mathrm{Cy} 5.5}^{\mathrm{TG}}(c=0)}{I_{\mathrm{Tb}}^{\mathrm{TG}}(c=0)}
$$

The concentration-dependent $F_{\mathrm{R}-\mathrm{BG}}$ values were used to determine the calibration curves for each miRNA (or ssDNA) target in each detection window. The slopes of the four linearly fitted calibration curves $\left(z_{20 \mathrm{a}}^{\mathrm{TG} 1}, z_{21}^{\mathrm{TG} 1}, z_{20 \mathrm{a}}^{\mathrm{TG} 2}, z_{21}^{\mathrm{TG} 2}\right)$ were used to determine the concentrations in the duplexed assays that led to the concentration recovery. Equation 4 was used to determine the target concentrations of miR-20a and miR-21 (or the ssDNA analogues) in the duplexed assays.

$$
\left[\begin{array}{l}
F_{\mathrm{R}-\mathrm{BG}}^{1} \\
F_{\mathrm{R}-\mathrm{BG}}^{2}
\end{array}\right]=\left[\begin{array}{l}
c(\mathrm{miR}-20 \mathrm{a}) \\
c(\mathrm{miR}-21)
\end{array}\right] \times\left[\begin{array}{cc}
z_{20 \mathrm{a}}^{\mathrm{TG}} & z_{21}^{\mathrm{TG} 1} \\
z_{20 \mathrm{a}}^{\mathrm{TG} 2} & z_{21}^{\mathrm{TG} 2}
\end{array}\right]
$$

where $F_{\mathrm{R}-\mathrm{BG}}{ }^{1}$ and $F_{\mathrm{R}-\mathrm{BG}}{ }^{2}$ are the background-subtracted FRET ratios in TG PL detection windows $\mathrm{TG}_{1}$ and $\mathrm{TG}_{2}$, respectively. 


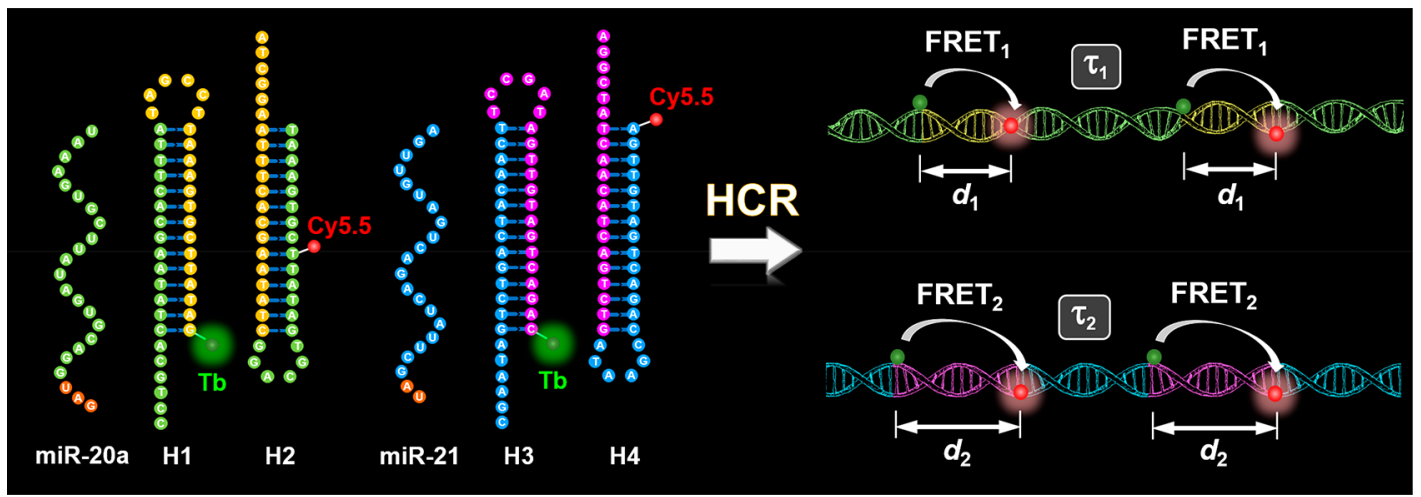

Figure 2. Schematic representation for duplexed (miR-20a and miR-21) detection of miRNAs using HCR-TG-FRET. Addition of targets to Tb and Cy5.5 labeled hairpin probes ( $\mathrm{H} 1$ to $\mathrm{H} 4$ ), which cannot engage in FRET due to their spatial separation, leads to opening of $\mathrm{H} 1$ and $\mathrm{H} 3$, which, in turn, open $\mathrm{H} 2$ and $\mathrm{H} 4$. This hairpin opening process triggers $\mathrm{HCR}$ because the open $\mathrm{H} 2$ and $\mathrm{H} 4$ possess the same sequences as the targets. Thus, long dsDNA concatemers are formed (right). In these dsDNA HCR products, $\mathrm{Tb}$ and $\mathrm{Cy} 5.5$ are in close proximity and can engage in FRET. The different distances in the two different HCR products $\left(d_{1}\right.$ and $\left.d_{2}\right)$ lead to different PL decays $\left(\tau_{1}\right.$ and $\left.\tau_{2}\right)$ because FRET is distance-dependent (cf. eqs 5 a and $5 b)$. See also Figure S1 for more details concerning the step by step procedure of HCR.

Gel Electrophoresis. Hairpin probes $\mathrm{H} 1(0.9 \mu \mathrm{M})$ and $\mathrm{H} 2$ $(0.9 \mu \mathrm{M})$ were heated to $95^{\circ} \mathrm{C}$ for 2 min and cooled slowly at room temperature for at least $2 \mathrm{~h}$ to form the hairpin structure before use. A $10 \mu \mathrm{L}$ portion of $\mathrm{H} 1$ and $10 \mu \mathrm{L}$ of target miRNA20a of different concentrations were placed in $0.5 \mathrm{~mL}$ Eppendorf tubes. After incubation at room temperature for $30 \mathrm{~min}$, a $10 \mu \mathrm{L}$ portion of $\mathrm{H} 2$ was added and the mixture was further incubated for $60 \mathrm{~min}$. SYBR green II (Thermo Fisher) was used as DNA stain. An $8 \%$ polyacrylamide gel was prepared with $30 \%$ acrylamide/bis-acrylamide solution (37.5:1, Catalog No. 1610158, Bio-Rad) and $1 \times$ TBE buffer ( $\mathrm{pH} 8.0$ ). A $15 \mu \mathrm{L}$ portion of each sample was loaded into the lane, and electrophoresis was performed on a Mini-PROTEAN Tetra electrophoresis system (Bio-Rad) at a constant potential of 200 $\mathrm{V}$ for $30 \mathrm{~min}$ with $0.5 \times \mathrm{TBE}$ ( $\mathrm{pH} 8.0$ ) as running buffer. The gel was then visualized under UV light.

\section{RESULTS AND DISCUSSION}

Principle of HCR-TG-FRET. The principle of enzyme-free duplexed miRNA detection based on a single Tb-Cy5.5 FRET pair and HCR is shown in Figure 2. Hairpin probes $\mathrm{H} 1$ and $\mathrm{H} 3$ were labeled with $\mathrm{Tb}$ FRET donors, whereas hairpin probes $\mathrm{H} 2$ and $\mathrm{H} 4$ were labeled with Cy5.5 FRET acceptors (internally for $\mathrm{H} 2$ and terminally for $\mathrm{H} 4$ ). The system $\mathrm{H} 1 / \mathrm{H} 2$ was designed for miR-20a and the system $\mathrm{H} 3 / \mathrm{H} 4$ for miR-21 detection. $\mathrm{H} 1$ and $\mathrm{H} 2$ ( $\mathrm{H} 3$ and $\mathrm{H} 4$ ) have 14 base pair (bp) stems, 6 nucleotide (nt) loops, and 6 nt sticky ends. Complementary sequences between $\mathrm{H} 1$ and $\mathrm{H} 2$ ( $\mathrm{H} 3$ and $\mathrm{H} 4)$ are shown in identical colors. The $6 \mathrm{nt}$ sticky end of $\mathrm{H} 1$ (H3) is complementary to the loop of $\mathrm{H} 2$ (H4), and the 6 nt sticky end of $\mathrm{H} 2$ (H4) is complementary to the loop of $\mathrm{H} 1(\mathrm{H} 3)$. In the absence of targets, all probes are stable and adopt hairpin structures. Thus, $\mathrm{Tb}$ and $\mathrm{Cy} 5.5$ are separated and cannot engage in FRET. In the presence of targets, which are complementary to $20 \mathrm{nt}$ ( $14 \mathrm{nt}$ from the stem plus $6 \mathrm{nt}$ from the sticky end) of $\mathrm{H} 1$ ( $\mathrm{H} 3)$, an unbiased stranddisplacement reaction opens the hairpin $\mathrm{H} 1(\mathrm{H} 3)$. The newly exposed ssDNA sequence of $\mathrm{H} 1(\mathrm{H} 3)$ will then be able to open hairpin $\mathrm{H} 2(\mathrm{H} 4)$. The consequently exposed ssDNA sequence of $\mathrm{H} 2(\mathrm{H} 4)$ is identical with the target sequence, which triggers the HCR that forms a long dsDNA concatemer composed of $\mathrm{H} 1$ and $\mathrm{H}_{2}\left(\mathrm{H}_{3}\right.$ and $\left.\mathrm{H}_{4}\right)$. Because $\mathrm{H} 1$ and $\mathrm{H}_{2}\left(\mathrm{H}_{3}\right.$ and $\left.\mathrm{H}_{4}\right)$ are conjugated with $\mathrm{Tb}$ and Cy5.5, many of these FRET pairs are brought into close proximity. Thus, HCR produces an amplified
TG-FRET signal. Another important feature of our HCR-TGFRET system is the difference in Tb to Cy5.5 distances in the $\mathrm{H} 1 / \mathrm{H} 2$ and $\mathrm{H} 3 / \mathrm{H} 4$ concatemers, respectively. Due to the internal labeling of $\mathrm{H} 2$ with Cy5.5, the Tb-Cy5.5 distance $\left(d_{1}\right)$ in the $\mathrm{H} 1 / \mathrm{H} 2 \mathrm{HCR}$ product is significantly closer than that in the H3/H4 HCR product $\left(d_{2}\right)$.

FRET efficiency $\left(E_{\text {FRET }}\right)$ is proportional to the inverse sixth power of the donor-acceptor distance, and the higher the $E_{\mathrm{FRET}}$, the stronger the quenching of the donor PL decay time $\left.\left(\tau_{\mathrm{D}}\right)\right)^{43,44}$ Thus, the longer distance $d_{2}$ will cause a lower FRET efficiency and a longer FRET-quenched donor PL decay time $\left(\tau_{\mathrm{DA}}\right)$ in comparison to the shorter distance $d_{1}$. Owing to the ca. $10^{6}$-fold difference between the PL lifetimes of $\mathrm{Tb}$ (approximately milliseconds) and Cy5.5 (approximately nanoseconds), the FRET-sensitized lifetime of the Cy5.5 acceptor $\left(\tau_{\mathrm{AD}}\right)$ is the same as the FRET-quenched lifetime of the Tb donor $\left(\tau_{\mathrm{AD}}=\tau_{\mathrm{DA}}\right){ }^{45}$ These relations among FRET efficiency, distance, and PL decay times are summarized in eq $5 \mathrm{a}$ for distance $d_{1}$ and eq $5 \mathrm{~b}$ for distance $d_{2}{ }^{43-45}$

$$
\begin{aligned}
& E_{\mathrm{FRET}_{1}}=\frac{R_{0}^{6}}{R_{0}^{6}+d_{1}^{6}}=1-\frac{\tau_{\mathrm{DA} 1}}{\tau_{\mathrm{D}}}=1-\frac{\tau_{\mathrm{AD} 1}}{\tau_{\mathrm{D}}} \\
& E_{\mathrm{FRET}_{2}}=\frac{R_{0}^{6}}{R_{0}^{6}+d_{2}^{6}}=1-\frac{\tau_{\mathrm{DA} 2}}{\tau_{\mathrm{D}}}=1-\frac{\tau_{\mathrm{AD} 2}}{\tau_{\mathrm{D}}}
\end{aligned}
$$

MicroRNA-Specific HCR-TG-FRET. To confirm both the target-specific formation of luminescent HCR products and the different $\mathrm{Tb}-\mathrm{Cy} 5.5$ donor-acceptor distances inside the two different HCR products (for miR-20a and miR-21), we performed gel electrophoresis and time-resolved PL spectroscopy. The gels (performed for miR-20a only) showed two main bands, one for the hairpin probes and another for the HCR products (Figure 3). For single-hairpin probes without target (first two lanes), no HCR product could be observed, and for the mixed-hairpin probes (third lane), only a very weak HCR product band appears (nonspecific opening of the hairpin probes). When increasing target concentrations were added to the mixed-hairpin probes, the HCR bands became clearly visible whereas the intensity of the hairpin probe bands got weaker with increasing target concentration because more $\mathrm{H} 1$ and $\mathrm{H} 2$ was consumed to form dsDNA structures (HCR products) with higher molecular weight. The formation of dsDNA structures of 


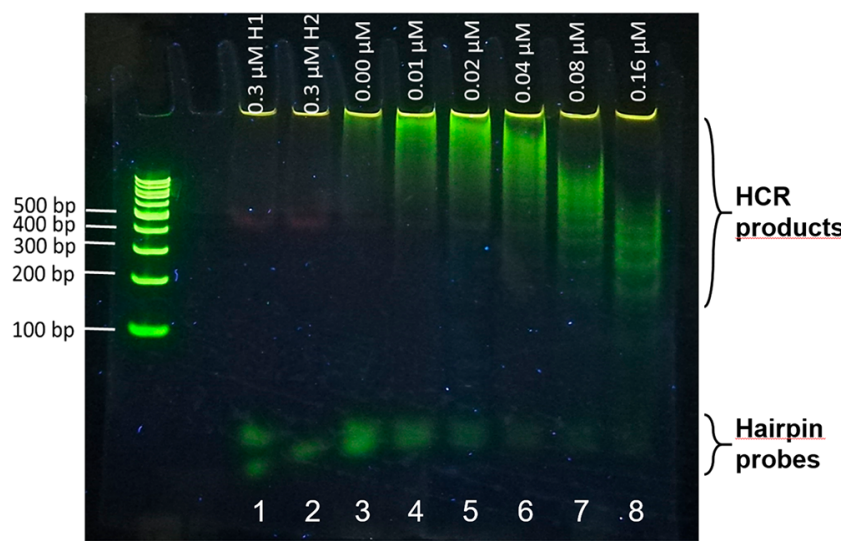

Figure 3. Electrophoresis gels for single hairpins ( $\mathrm{H} 1$ in lane 1 and $\mathrm{H} 2$ in lane 2), mixed hairpins ( $\mathrm{H} 1$ and $\mathrm{H} 2$ in lane 3), and mixed hairpins with increasing target (miR-20a) concentrations $(0.01-0.16 \mu \mathrm{M}$ in lanes 4-8). Hairpin probe bands are split due to open and closed probes.

different lengths (different molecular weights) resulted in a relatively broad smearing of the HCR bands. At the highest concentration $(0.16 \mu \mathrm{M})$, different bands became clearly visible, indicating the formation of shorter dsDNA HCR products. The availability of many targets at such high target concentrations led to the formation of many assembly sites and a lower probability for $\mathrm{H} 1$ and $\mathrm{H} 2$ to assemble into longer structures. ${ }^{3,46}$

Time-resolved PL curves of FRET-sensitized Cy5.5 at different target concentrations (between 0.01 and $0.7 \mathrm{nM}$ ) clearly showed the different decay times caused by the different Tb-Cy5.5 distances (Figure 4). Owing to the shorter distance $\left(d_{1}\right.$ in Figure 2), miR-20a-specific HCR products led to significantly faster decays $\left(\tau_{\mathrm{DA}}=360 \pm 10 \mu \mathrm{s}\right)$ in comparison to the miR-21-specific HCR products $\left(\tau_{\mathrm{DA}}=930 \pm 50 \mu \mathrm{s}\right)$. These average PL decay times resulted in FRET efficiencies of $E_{\mathrm{FRET}}(\mathrm{miR}-20 \mathrm{a})=0.87 \pm 0.01$ and $E_{\mathrm{FRET}}(\mathrm{miR}-21)=0.66 \pm 0.02$ and distances of $d_{1}=4.3 \pm 0.1 \mathrm{~nm}$ and $d_{2}=5.2 \pm 0.2 \mathrm{~nm}$ (as calculated by eqs $5 \mathrm{a}$ and $5 \mathrm{~b}$ with $R_{0}=5.8 \pm 0.2 \mathrm{~nm}$; cf. Materials and Methods). On the basis of a distance of $\sim 0.33 \mathrm{~nm}$ between two bp within a dsDNA ${ }^{47}$ and the 12 (miR-20a-specific HCR product) and 20 (miR-21-specific HCR product) bp Tb-Cy5.5 separation, the expected distances would be 4.0 and $6.6 \mathrm{~nm}$. Taking into account the differences in Cy5.5-DNA conjugation (internal labeling for $\mathrm{H} 2$ and terminal labeling for $\mathrm{H} 4$ ), the flexible linkers between the DNA and the fluorophores, and the many nicks inside the long dsDNA HCR products, these values are in relatively good agreement with the measured average distances $d_{1}$ and $d_{2}$. The most important property for our multiplexing approach was the significant difference between the two decay times (caused by different distances), and the exact reason for the differences in expected and measured donoracceptor distances is of minor relevance. The decay curves for both miRNA targets also show increasing intensities with increasing target concentrations, which confirms the concentration-dependent HCR production and the possibility to use TG-FRET for miRNA quantification.

LODs, Sensitivity, and Specificity. The diagnostics performance of the miR-20a and miR-21 HCR-TG-FRET assays was evaluated on a clinical fluorescence plate reader (KRYPTOR) concerning LODs (lowest detectable concentration, i.e., 3 standard deviations over background), sensitivity (slope of signal over concentration), and specificity (distinction of specific target from others). The assay calibration curves (Figure 5A,B) showed linearly increasing time-gated FRET ratios (intensity ratio of $\mathrm{Cy} 5.5$ and $\mathrm{Tb} \mathrm{PL}$ in a time window from 0.1 to $0.9 \mathrm{~ms}$ after the excitation pulse) with increasing concentrations from 0.005 to $0.3 \mathrm{nM}$ for miR-20a and from 0.005 to $0.25 \mathrm{nM}$ for miR-21. This linear dynamic range can be further increased by increasing the hairpin concentrations (Figure S6). Even higher concentrations may be quantified by using the automatic dilution mode of the KRYPTOR system. The calibration curves, which show the target concentrations in the $140 \mu \mathrm{L}$ detection volumes in the 96-well microtiter plates, revealed LODs (standard deviation was calculated for 30 independent background measurements, which contained all assay components but no miRNA target) of $2.8 \mathrm{pM}(390 \mathrm{amol})$ of miR-20a and $1.7 \mathrm{pM}(240 \mathrm{amol})$ of miRNA-21. The sensitivities were $(1.64 \pm 0.02) \times 10^{-4} F_{R} / p M$ for miR-20a and $(5.7 \pm 0.1) \times 10^{-5} F_{R} / p M$ for miR-21. Taking into account the rather small standard deviations (e.g., background standard deviations of $1.3 \times 10^{-4} F_{R}$ for miR-20a and $7.7 \times 10^{-5} F_{R}$ for miR-21) and the excellent fit of the data points to the linear calibration curves, this sensitivity allows distinguishing concentration differences of a few picomolar over the entire dynamic concentration range.

The target specificity of HCR-TG-FRET was evaluated for miR-20a and miR-21 (Figure 5C,D) against each other and against miR-20b, which possesses only two nucleotide
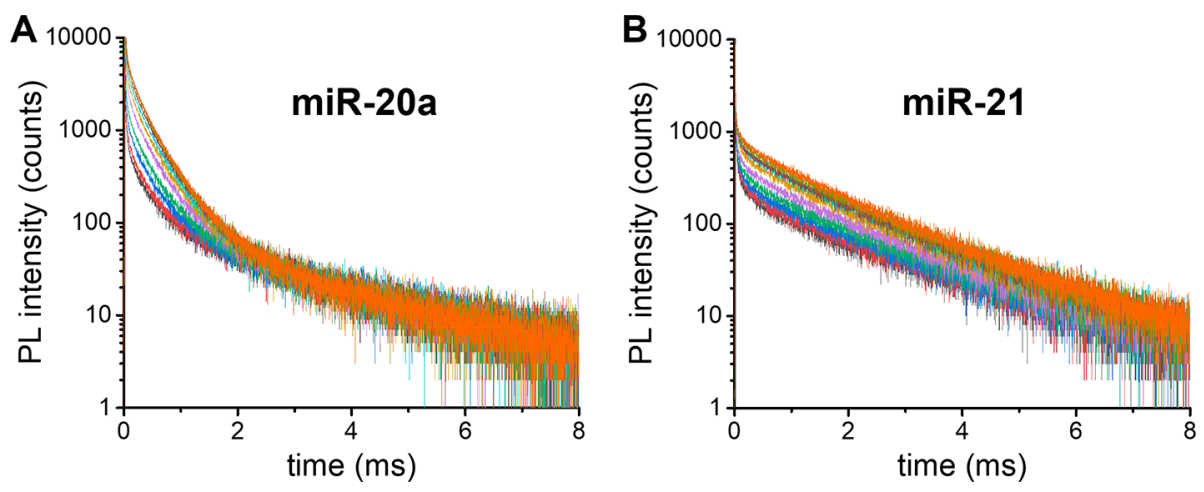

Figure 4. PL decay curves of the Cy5.5 FRET acceptor (Tb FRET donor curves are shown in Figure S2) for miR-20a (A) and miR-21 (B) detection at increasing target concentrations $(0,0.01,0.05,0.1,0.2,0.3,0.4,0.5,0.6$, and $0.7 \mathrm{nM}$ from black to orange $)$ and constant hairpin concentrations $(2 \mathrm{nM})$. Average PL decay times were $\tau_{\mathrm{DA}}=360 \pm 10 \mu \mathrm{s}$ for miR-20a and $\tau_{\mathrm{DA}}=930 \pm 50 \mu \mathrm{s}$ for miR-21 (cf. Figures S3 and S4 for calculation). PL decay curves for DNA targets are shown in Figure S5. 

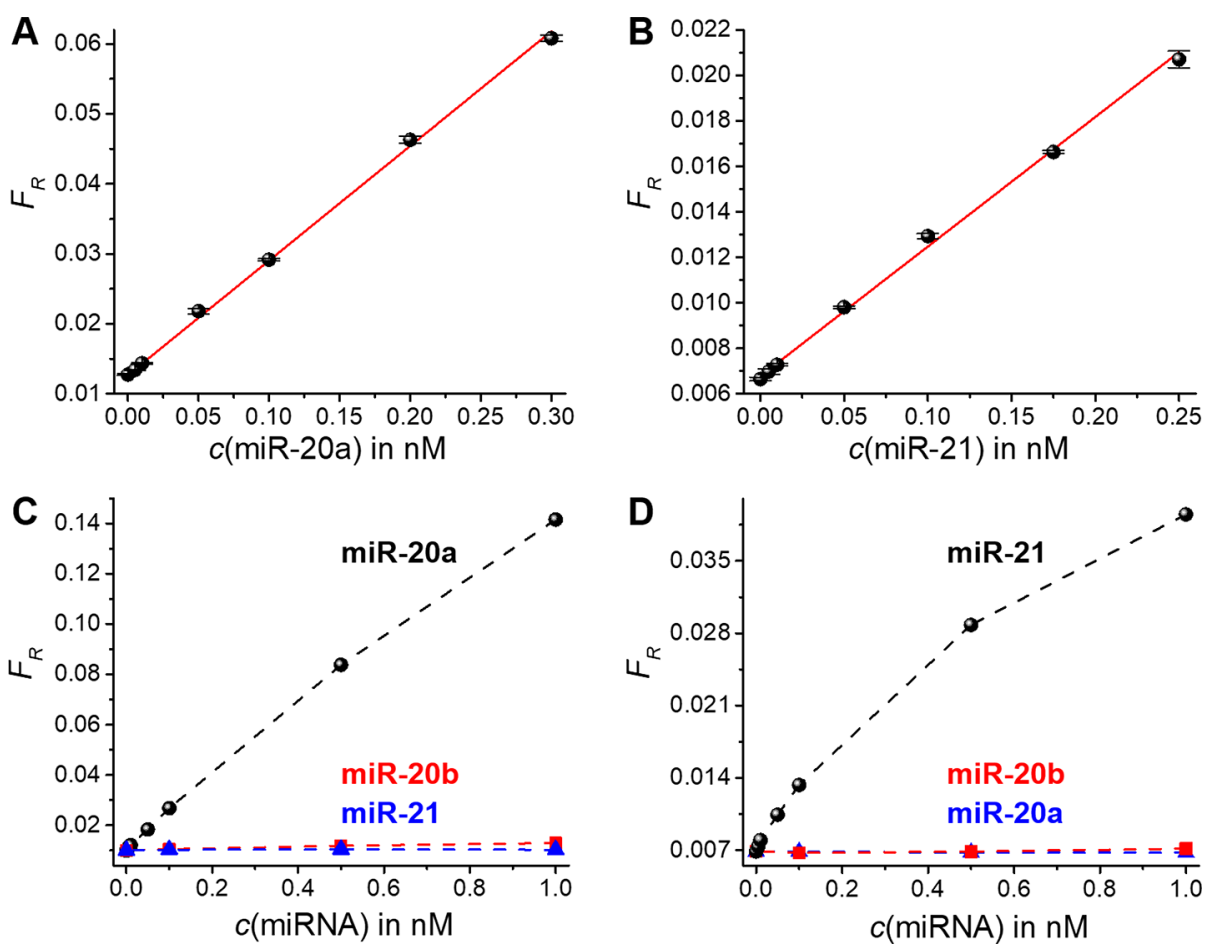

Figure 5. Diagnostic performance evaluation of the single miRNA sensors. Assay calibration curves (A for miR-20a and B for miR-21) showed a linearly increasing FRET ratio over target concentration with LODs of $2.8 \mathrm{pM}$ (390 amol) of miR-20a and $1.7 \mathrm{pM}(240 \mathrm{amol})$ of miRNA-21. Error bars present standard deviations from 30 measurements for background (no target, $c=0$ ) and 9 measurements for all other data points. Calibration curves for DNA targets are shown in Figure S7. Target specificity (C for miR-20a and D for miR-21) was demonstrated against two different miRNA targets.

mismatches (one at the $5^{\prime}$-terminus and the other in the center) in comparison to miR-20a (cf. Table 1). Both sensors (for miR20a and miR-21) showed concentration-dependent increasing FRET ratios exclusively for their target, whereas the other two targets led to background signals over the entire concentration range. Only at the highest concentration of miR-20b $(1 \mathrm{nM}) \mathrm{did}$ the miR-20a sensor show a minor increase of the FRET ratio in comparison to the background signal. Overall, HCR-TG-FRET quantification of miRNA provided excellent performance concerning limits of detection, sensitivity, and specificity.

Single FRET-Pair Multiplexing. TG multiplexing from a single measurement requires the efficient distinction of different TG detection windows. We selected two TG windows and recorded calibration curves for both miRNA targets (Figure $6 \mathrm{~A}, \mathrm{~B})$, whose slopes are required for quantification of both targets from the same sample (eq 4, Materials and Methods). The background-corrected FRET ratios of both TG windows for both targets increased linearly until $0.15 \mathrm{nM}$ for miR-20a and $0.30 \mathrm{nM}$ for miR-21. Due to the faster PL decay of the Tb-Cy5.5 FRET pair in the miR-20a HCR-TG-FRET sensor, the PL intensity in the second $\mathrm{TG}$ window $\left(\mathrm{TG}_{2}\right)$ is almost exclusively dependent on miR-21 concentration. On the other hand, the miR-21 sensitivity in $\mathrm{TG}_{2}$ (slope of the blue calibration curve in Figure $6 \mathrm{~B}$ ) is ca. 4 times lower than the miR-20a sensitivity in $\mathrm{TG}_{1}$ (slope of the green calibration curve in Figure 6A). Taking into account the ca. 2-fold lower sensitivity of miR-21 in comparison to miR-20a in $\mathrm{TG}_{1}$ (slopes of the green calibration curves in Figure 6A,B), it can be expected that miR-20a can be measured with higher precision in a duplexed assay. Such considerations are important for multiplexed assay design because different targets often require different sensitivities for diagnostic evaluation.
A first experiment to demonstrate duplexed quantification of miR-20a and miR-21 from the same sample by a single measurement consisted of mixing both targets at the same increasing concentrations that were used for the single-sensor assays (0-0.15 nM for miR-20a and $0-0.3 \mathrm{nM}$ for miR-21). As expected, the PL decay curves presented a multiexponential convolution of the single curves and the FRET ratios increased in both TG detection windows (Figure 6C). The almost perfect overlap of the detected FRET ratios and the sum of the singleassay FRET ratios (Figure 6C, right) showed that a combination of duplexed FRET ratios and the slopes of the single-assay calibration curves (eq 4) should be applicable to simultaneously quantify different concentrations of miR-20a and miR-21 from the same sample. To verify this finding, we prepared 13 samples that contained both targets at different concentrations ranging from high miR-20a and low miR-21 to low miR-20a and high miR-21. As shown in Figure 7, both miRNA targets could be quantified at varying concentrations between 30 and $300 \mathrm{pM}$ with relatively high precision. The performance of HCR-TGFRET was not compromised by the addition of serum (10\%) inside the sample (Figure 7 and Figure S10), and RNase inhibitors were not required. As expected, due to the lower sensitivity of the miR-21 sensor (vide supra), the accuracy of miR-21 quantification was lower. However, all experimentally determined data points are still very close to the known concentrations. Taking into account that all measured miR-21 concentrations have a slightly less steep linear correlation with the actual concentrations (as visualized by the blue dashed lines in Figure 7), this deviation may even be correctable in case a higher accuracy should be required. 

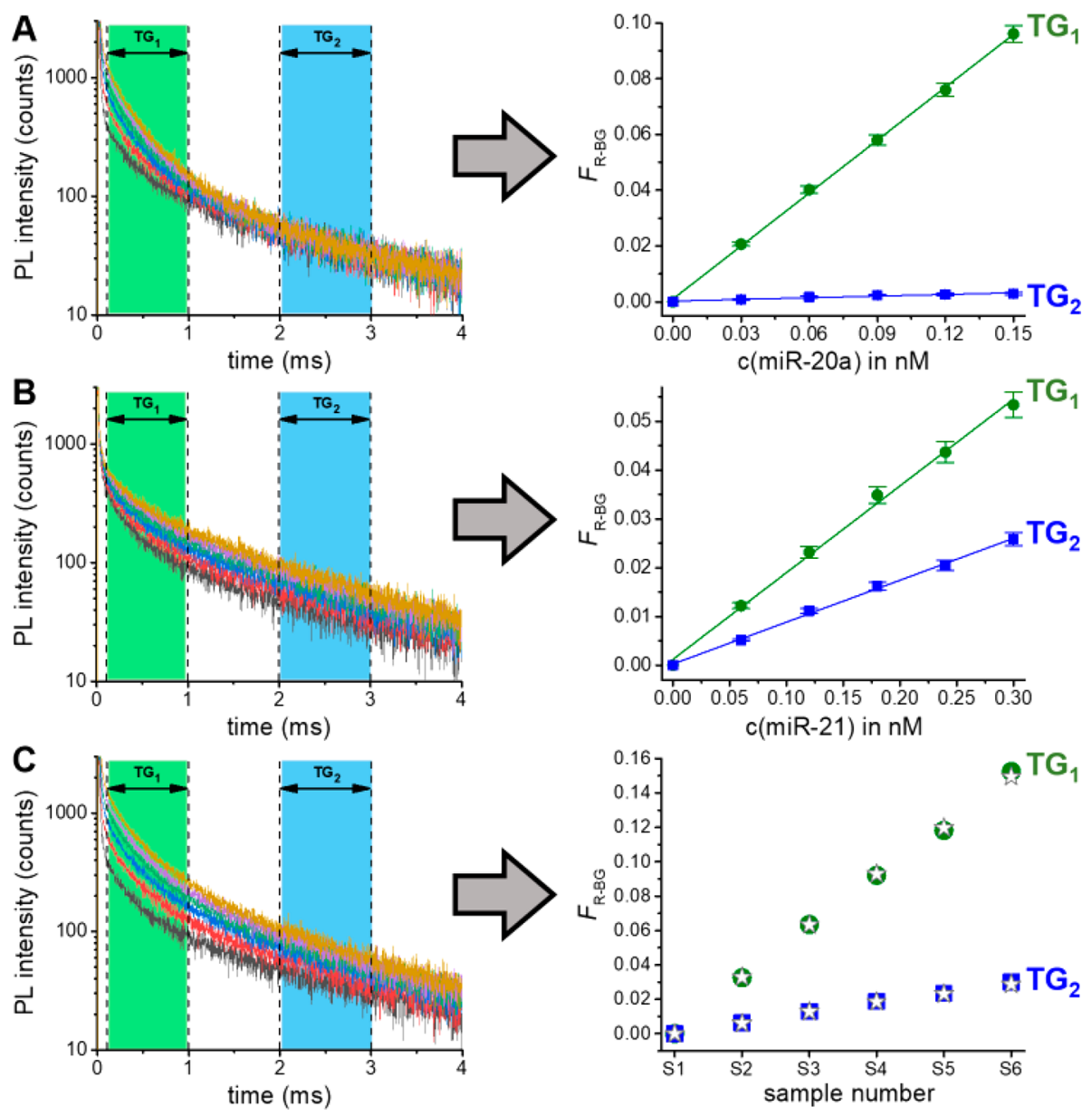

Figure 6. PL decay curves of FRET-sensitized Cy5.5 (PL decay curves of Tb are shown in Figure S8) and corresponding FRET ratios (right) determined from the TG PL intensities in the two TG detection windows: (A) miR-20a (error bars present the maximum relative deviation from the linear fit: $3.1 \%$ for $\mathrm{TG}_{1}$ and $16 \%$ for $\mathrm{TG}_{2}$ ); (B) miR-21 (error bars present the maximum relative deviation from the linear fit: $4.9 \%$ for $\mathrm{TG}_{1}$ and $5.2 \%$ for $\mathrm{TG}_{2}$ ); (C) miR-20a and miR-21. Stars in (C) present the mathematical sum of the FRET ratios from the single miRNA-target assays in (A) and (B). The overlap with the measured duplexed FRET ratios shows the applicability of the calibration curves from (A) and (B) to duplexed miRNA quantification in (C). Decay and calibration curves for DNA targets are shown in Figure S9.
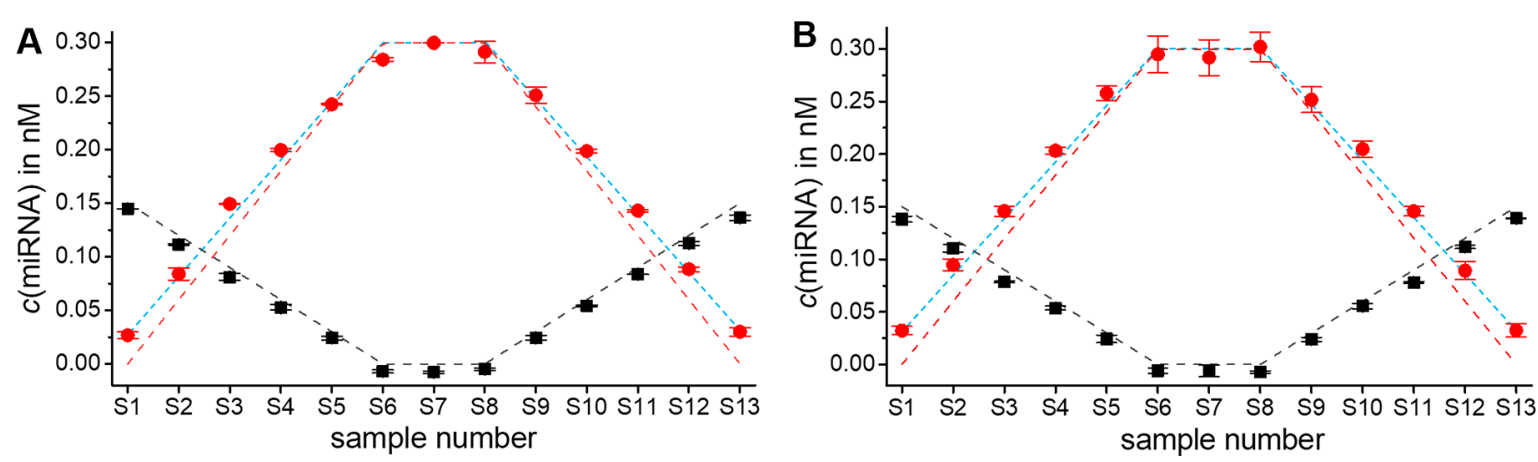

Figure 7. Duplexed HCR-TG-FRET quantification of miR-20a (black) and miR-21 (red) at varying concentrations in buffer (A) and in buffer containing 10\% serum (B). Dashed lines represent known concentrations. Data points were determined by HCR-TG-FRET using the calibration curves from Figure 6 and eq 4. The light blue dashed shows the slope of the red data points, which may be used to further improve the accuracy for miR21. Duplexed detection of DNA targets is shown in Figure S11.

\section{CONCLUSION}

HCR is a very simple signal amplification strategy because it does not require any enzymes. ${ }^{2}$ Therefore, it is a frequently applied technique for sensitive nucleic acid detection. Although HCR has been significantly optimized since its first application in 2004, , $^{72-15}$ we showed that simplicity, sensitivity, and multiplexing can be further improved by implementation of TG-
FRET into HCR. Because FRET can only occur in the HCR product and not in the free hairpin probes, HCR-TG-FRET does not require any washing or separation steps (simplicity improvement). The TG detection of $\mathrm{Tb}$ donor and Cy5.5 acceptor with long PL decay times allowed for efficient suppression of sample autofluorescence and of directly excited acceptor fluorescence, which leads to high signal to background 
ratios (sensitivity improvement). Using different TG detection windows enabled multiplexed detection of different targets from the same sample by a single measurement, single excitation wavelength, and single detector (multiplexing and simplicity improvement). We applied HCR-TG-FRET to both miRNA (miR-20a and miR-21) and the analogous ssDNA targets and demonstrated very low LODs (down to $240 \mathrm{amol}$ of miRNA and 123 amol of ssDNA) with high sensitivities. High specificity was verified against other targets, including very homologous miRNAs (miR-20a vs miR-20b). Single-measurement multiplexing was demonstrated by simultaneous quantification of miR-20a and miR-21 at different concentrations (between 30 and $300 \mathrm{pM}$ ) from the same sample with similar results for serum-free buffer and buffer containing $10 \%$ of serum without the use of RNase inhibitors. Our detailed analytical evaluation revealed the versatile and powerful diagnostic performance of HCR-TG-FRET even in challenging biological environments. Taking into account that the temporal multiplexing approach is not limited to two TG detection windows ${ }^{26}$ and that it can be combined with spectral distinction for higher order multiplexing, ${ }^{27}$ we expect that HCR-TG-FRET will become a very useful alternative to currently applied HCR approaches for advanced multiplexed nucleic acid detection both in solution and in situ.

\section{ASSOCIATED CONTENT}

\section{S Supporting Information}

The Supporting Information is available free of charge on the ACS Publications website at DOI: 10.1021/acs.analchem.8b05600.

Schematic presentations of the HCR process and the principle of duplexed HCR-FRET for the detection of two targets, additional PL decay curves (of Cy5.5 and $\mathrm{Tb}$ ) at varying miRNA target concentrations, PL decay fits and distance determination, assay calibration curves for miRNA targets at higher hairpin concentrations, FRET ratio comparison between serum-free and serumcontaining samples, PL decay curves (of Cy5.5 and $\mathrm{Tb}$ ) at varying DNA target concentrations, diagnostic performance evaluation of the DNA assays, and duplexed quantification of ssDNA-20a and ssDNA-21 by HCRTG-FRET (PDF)

\section{AUTHOR INFORMATION}

\section{Corresponding Author}

*E-mail for N.H.: niko.hildebrandt@u-psud.fr.

\section{ORCID}

Niko Hildebrandt: 0000-0001-8767-9623

Notes

The authors declare no competing financial interest.

\section{ACKNOWLEDGMENTS}

We thank Lumiphore, Inc. for the gift of Lumi4® reagents. This work was supported by the French National Research Agency (ANR projects "PhenX" and "AMPLIFY"), the Institut Universitaire de France (IUF), the China Scholarship Council (CSC), and the European Commission (Horizon 2020, FETOpen project PROSEQO).

\section{REFERENCES}

(1) Zhao, Y. X.; Chen, F.; Li, Q.; Wang, L. H.; Fan, C. H. Chem. Rev. 2015, 115, 12491-12545.
(2) Dirks, R. M.; Pierce, N. A. Proc. Natl. Acad. Sci. U. S. A. 2004, 101, 15275-15278.

(3) Chen, Y.; Chen, L.; Ou, Y. D.; Guo, L. Q.; Fu, F. F. Sens. Actuators, B 2016, 233, 691-696.

(4) Shi, X. M.; Fan, G. C.; Tang, X. Y.; Shen, Q. M.; Zhu, J. J. Biosens. Bioelectron. 2018, 109, 190-196.

(5) Yang, L.; Liu, C. H.; Ren, W.; Li, Z. P. ACS Appl. Mater. Interfaces 2012, 4, 6450-6453.

(6) Ge, Z. L.; Lin, M. H.; Wang, P.; Pei, H.; Yan, J.; Sho, J. Y.; Huang, Q.; He, D. N.; Fan, C. H.; Zuo, X. L. Anal. Chem. 2014, 86, 2124-2130.

(7) Choi, H. M. T.; Chang, J. Y.; Trinh, L. A.; Padilla, J. E.; Fraser, S. E.; Pierce, N. A. Nat. Biotechnol. 2010, 28, 1208-U103.

(8) Huang, J.; Wang, H.; Yang, X. H.; Quan, K.; Yang, Y. J.; Ying, L.; Xie, N. L.; Ou, M.; Wang, K. M. Chem. Sci. 2016, 7, 3829-3835.

(9) Li, X. Y.; Li, J. J.; Zhu, C. X.; Zhang, X. H.; Chen, J. H. Talanta 2018, 182, 292-298.

(10) Lin, R.; Feng, Q. R.; Li, P.; Zhou, P.; Wang, R. Y.; Liu, Z.; Wang, Z. Q.; Qi, X. B.; Tang, N.; Shao, F.; Luo, M. M. Nat. Methods 2018, 15, 275-278.

(11) Zhu, G. Z.; Zhang, S. F.; Song, E. Q.; Zheng, J.; Hu, R.; Fang, X. H.; Tan, W. H. Angew. Chem., Int. Ed. 2013, 52, 5490-5496.

(12) Choi, H. M. T.; Calvert, C. R.; Husain, N.; Huss, D.; Barsi, J. C.; Deverman, B. E.; Hunter, R. C.; Kato, M.; Lee, S. M.; Abelin, A. C. T.; Rosenthal, A. Z.; Akbari, O. S.; Li, Y. W.; Hay, B. A.; Sternberg, P. W.; Patterson, P. H.; Davidson, E. H.; Mazmanian, S. K.; Prober, D. A.; van de Rijn, M.; Leadbetter, J. R.; Newman, D. K.; Readhead, C.; Bronner, M. E.; Wold, B.; Lansford, R.; Sauka-Spengler, T.; Fraser, S. E.; Pierce, N. A. Development 2016, 143, 3632-3637.

(13) Choi, H. M. T.; Schwarzkopf, M.; Fornace, M. E.; Acharya, A.; Artavanis, G.; Stegmaier, J.; Cunha, A.; Pierce, N. A. Development 2018, 145, dev165753.

(14) Rana, M.; Balcioglu, M.; Kovach, M.; Hizir, M. S.; Robertson, N. M.; Khan, I.; Yigit, M. V. Chem. Commun. 2016, 52, 3524-3527.

(15) Choi, H. M. T.; Beck, V. A.; Pierce, N. A. ACS Nano 2014, 8, 4284-4294.

(16) Geissler, D.; Stufler, S.; Lohmannsroben, H. G.; Hildebrandt, N. J. Am. Chem. Soc. 2013, 135, 1102-1109.

(17) Jin, Z. W.; Geissler, D.; Qiu, X.; Wegner, K. D.; Hildebrandt, N. Angew. Chem., Int. Ed. 2015, 54, 10024-10029.

(18) Bao, B. Q.; Zhu, J.; Gong, L. N.; Chen, J.; Pan, Y. R.; Wang, L. H. RSC Adv. 2017, 7, 3528-3533.

(19) Li, J.; Li, D. X.; Yuan, R.; Xiang, Y. ACS Appl. Mater. Interfaces 2017, 9, 5717-5724.

(20) Ou, M.; Huang, J.; Yang, X. H.; He, X. X.; Quan, K.; Yang, Y. J.; Xie, N. L.; Li, J.; Wang, K. M. ChemBioChem 2018, 19, 147-152.

(21) Quan, K.; Huang, J.; Yang, X. H.; Yang, Y. J.; Ying, L.; Wang, H.; Xie, N. L.; Ou, M.; Wang, K. M. Anal. Chem. 2016, 88, 5857-5864.

(22) Wang, H. M.; Li, C. X.; Liu, X. Q.; Zhou, X.; Wang, F. Chem. Sci. 2018, 9, 5842-5849.

(23) Wei, J.; Gong, X.; Wang, Q.; Pan, M.; Liu, X. Q.; Liu, J.; Xia, F.; Wang, F. Chem. Sci. 2018, 9, 52-61.

(24) Zhang, S. Q.; Wang, K.; Li, K. B.; Shi, W.; Jia, W. P.; Chen, X. Y.; Sun, T.; Han, D. M. Biosens. Bioelectron. 2017, 91, 374-379.

(25) Quan, K.; Li, J.; Xie, N. L.; Wei, Q.; Tang, J.; Yang, X.; Wang, K.; Huang, J. Chem. Sci. 2019, DOI: 10.1039/C8SC04887H.

(26) Qiu, X.; Guo, J. J.; Jin, Z. W.; Petreto, A.; Medintz, I. L.; Hildebrandt, N. Small 2017, 13, 1700332.

(27) Qiu, X.; Guo, J. J.; Xu, J. Y.; Hildebrandt, N. J. Phys. Chem. Lett. 2018, 9, 4379-4384.

(28) Qiu, X.; Hildebrandt, N. ACS Nano 2015, 9, 8449-8457.

(29) Qiu, X.; Xu, J.; Guo, J.; Yahia-Ammar, A.; Kapetanakis, N.-I.; Duroux-Richard, I.; Unterluggauer, J. J.; Golob-Schwarzl, N.; Regeard, C.; Uzan, C.; Gouy, S.; DuBow, M.; Haybaeck, J.; Apparailly, F.; Busson, P.; Hildebrandt, N. Chem. Sci. 2018, 9, 8046-8055.

(30) CisBio, HTRF Technology: https://www.cisbio.com/usa/drugdiscovery/htrf-technology, November 2018.

(31) Zwier, J. M.; Hildebrandt, N.: Time-Gated FRET Detection for Multiplexed Biosensing. In Reviews in Fluorescence 2016; Geddes, C. D., Ed.; Springer International: Cham, Switzerland, 2017; pp 17-43. 
(32) Xie, B. Y.; Ding, Q.; Han, H. J.; Wu, D. Bioinformatics 2013, 29, 638-644.

(33) Xie, B.; Ding, Q. miRCancer database; http://mircancer.ecu. edu/index.jsp, November 2018.

(34) Ro, S.; Park, C.; Jin, J. L.; Sanders, K. M.; Yan, W. Biochem. Biophys. Res. Commun. 2006, 351, 756-763.

(35) Baker, M. Nat. Methods 2010, 7, 687-692.

(36) Zhou, Y. T.; Huang, Q.; Gao, J. M.; Lu, J. X.; Shen, X. Z.; Fan, C. H. Nucleic Acids Res. 2010, 38, No. e156.

(37) Deng, R. J.; Tang, L. H.; Tian, Q. Q.; Wang, Y.; Lin, L.; Li, J. H. Angew. Chem., Int. Ed. 2014, 53, 2389-2393.

(38) Dai, W. H.; Dong, H. F.; Guo, K. K.; Zhang, X. J. Chem. Sci. 2018, 9, 1753-1759.

(39) Qu, X. J.; Jin, H. J.; Liu, Y. Q.; Sun, Q. J. Anal. Chem. 2018, 90, $3482-3489$.

(40) Zhang, Y.; Zhang, C. Y. Anal. Chem. 2012, 84, 224-231.

(41) Jia, H. X.; Li, Z. P.; Liu, C. H.; Cheng, Y. Q. Angew. Chem., Int. Ed. 2010, 49, 5498-5501.

(42) Lumiprobe, Cyanine5.5 NHS ester: https://www.lumiprobe. com/p/cy55-nhs-ester, November 2018.

(43) FRET - Förster Resonance Energy Transfer. From Theory to Applications; Medintz, I. L., Hildebrandt, N., Eds.; Wiley-VCH: Weinheim, Germany, 2014.

(44) Lakowicz, J. R.: Principles of fluorescence spectroscopy, 3rd ed.; Springer: New York, 2006.

(45) Hildebrandt, N.; Wegner, K. D.; Algar, W. R. Coord. Chem. Rev. 2014, 273, 125-138.

(46) Yang, X. J.; Yu, Y. B.; Gao, Z. Q. ACS Nano 2014, 8, 4902-4907.

(47) Mandelkern, M.; Elias, J. G.; Eden, D.; Crothers, D. M. J. Mol. Biol. 1981, 152, 153-161. 